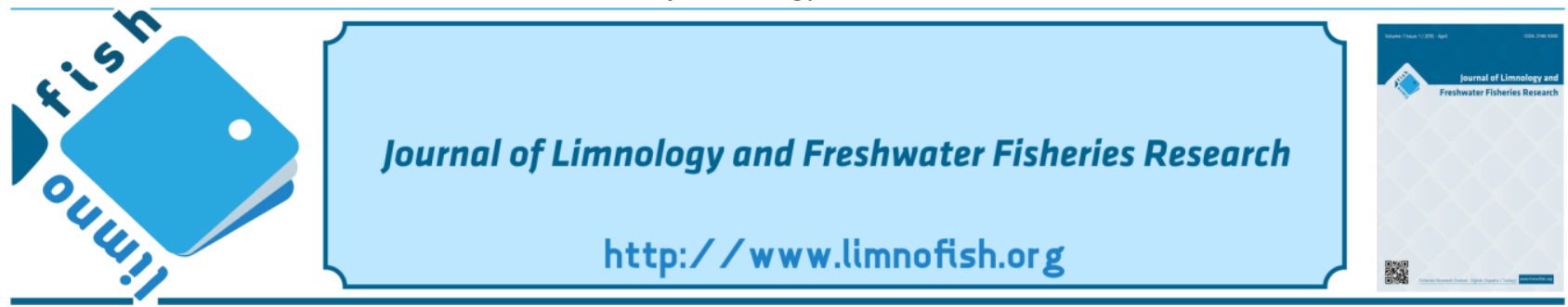

\title{
Zooplankton Fauna of Demrek Dam Lake (Kırıkan, Hatay)
}

\author{
Ahmet BOZKURT ${ }^{1 *}$ (D), Bestami KARA ${ }^{1}$ (iD \\ ${ }^{1}$ İskenderun Technical University, Faculty of Marine Sciences and Technology, İskenderun, Hatay, Turkey
}

\section{A B S T RACT}

The annual average of Secchi disk depth $(100.42 \pm 38.99 \mathrm{~cm})$, water temperature $\left(21.06 \pm 5.96^{\circ} \mathrm{C}\right)$, dissolved oxygen $(8.33 \pm 0.99 \mathrm{mg} / \mathrm{l})$, nitrate $(0.43 \pm 0.19 \mathrm{mg} / \mathrm{l})$, nitrite $(0.02 \pm 0.01)$, hardness $(172 \pm 17.27 \mathrm{mg} / \mathrm{l})$, silica $(1.17 \pm 0.28 \mathrm{mg} / \mathrm{l})$, phosphate $(0.14 \pm 0.03 \mathrm{mg} / \mathrm{l})$, organic phosphate $(1.09 \pm 0.56 \mathrm{mg} / \mathrm{l})$ and chlorophyll- $a$ $(0.05 \pm 0.03 \mathrm{mg} / \mathrm{l})$ were detected and according to these values, it was determined that the reservoir water has eutrophic and hyperneurophic character. Rotifera had the highest proportion with 45 taxa, followed by Cladocera with 11 species and Copepoda with 7 species. Asplanchna priodonta, Keratella cochlearis, Polyarthra dolichoptera, Sychaeta stylata Bosmina longirostris, Diaphanosoma birgei, and Disparalona rostrata were present throughout the whole study period. The most abundant species from Rotifera was Sychaeta stylata $(26034 \pm 56482.24$ ind. $\left./ \mathrm{m}^{3}\right)$ followed by Polyarthra dolichoptera $\left(15356 \pm 9593.48\right.$ ind. $\left./ \mathrm{m}^{3}\right)$ and Keratella cochlearis $\left(11850 \pm 15441.51\right.$ ind. $\left./ \mathrm{m}^{3}\right)$. In the study, the most common species belonging to Cladocera was Ceriodaphnia pulchella $(7042 \pm 6759.93$ ind. $/ \mathrm{m}^{3}$ ) and the most abundant copepod species was Cyclops vicinus $\left(2553 \pm 1596.48\right.$ ind. $\left./ \mathrm{m}^{3}\right)$.

\section{ARTICLE INFO}

\section{RESEARCH ARTICLE}

Received : 21.06 .2020

Revised : 24.08 .2020

Accepted : 30.08 .2020

Published : 29.12.2020

DOI:10.17216/LimnoFish.755863

\section{* CORRESPONDING AUTHOR}

ahmet.bozkurt@iste.edu.tr

Phone : +9032661416 93/3405

Keywords: Demrek Dam Lake, water quality, zooplankton

\section{Demrek Baraj Gölü (Kırıkhan, Hatay) Zooplankton Faunası}

Öz: Yıllık ortalama secchi disk derinliği $(100,42 \pm 38,99 \mathrm{~cm})$, su sıcaklığı $\left(21,06 \pm 5,96{ }^{\circ} \mathrm{C}\right)$, çözünmüş oksijen $(8,33 \pm 0,99 \mathrm{mg} /$ 1), nitrat $(0,43 \pm 0,19 \mathrm{mg} / 1)$, nitrit $(0,02 \pm 0,01)$, sertlik $(172 \pm 17,27 \mathrm{mg} / 1)$, silikat $(1,17 \pm 0,28 \mathrm{mg} / 1)$, fosfat $(0,14 \pm 0,03 \mathrm{mg} / 1)$, organik fosfat $(1,09 \pm 0,56 \mathrm{mg} / 1)$ ve klorofil-a $(0,05 \pm 0,03 \mathrm{mg} / 1)$ tespit edildi ve bu değerlere göre rezervuar suyunun ötrofik ve hiperneurofik karaktere sahip olduğu belirlendi. Rotifera 45 taksonla en yüksek orana sahipken, onu 11 tür ile Cladocera ve 7 tür ile Copepoda izledi. Bütün çalışma süresince Asplanchna priodonta, Keratella cochlearis, Polyarthra dolichoptera, Sychaeta stylata, Bosmina longirostris, Diaphanosoma birgei ve Disparalona rostrata mevcuttu. Rotifera'dan en bol bulunan tür Sychaeta stylata $\left(26034 \pm 56482,24\right.$ birey $\left./ \mathrm{m}^{3}\right)$ iken, bunu Polyarthra dolichoptera $\left(15356 \pm 9593,48\right.$ birey $\left./ \mathrm{m}^{3}\right)$ ve Keratella cochlearis $(11850 \pm$ 15441,51 birey $\left./ \mathrm{m}^{3}\right)$ takip etmiştir. Çalışmada Kladosera'ya ait en yaygın türün Ceriodaphnia pulchella $\left(7042 \pm 6759,93 \mathrm{birey} / \mathrm{m}^{3}\right)$ ve Kopepoda'ya ait en bol türün ise Cyclops vicinus $\left(2553 \pm 1596,48\right.$ birey $\left./ \mathrm{m}^{3}\right)$ olduğu belirlenmiş̧ir.

Anahtar kelimeler: Demrek Baraj Gölü, su kalitesi, zooplankton

\section{How to Cite}

Bozkurt A, Kara B. 2020. Zooplankton Fauna of Demrek Dam Lake (Kirikhan, Hatay). LimnoFish. 6(3): 189-200. doi: 10.17216/LimnoFish.755863

\section{Introduction}

The zooplanktonic organisms living in the lake ecosystem not only form the nutrients of planktivorous fish but also become a source of food for all insects, fish larvae, invertebrates, and other aquatic animals in the ecosystem. Besides, zooplankton are potential indicators for the water properties, pollution, and eutrophication status of the waters in which they are found (Hecky and Kilham 1973; Bērzinš and Pejler 1987; Mikschi 1989).
Various studies have reported that there is a close relationship between the efficiency of the aquatic environment and zooplanktonic organisms; since pollution has negative effects on zooplankton. In lake ecosystems, there is a balance between the living and inanimate factors of the lake.

Since the damages caused by people to nature disrupt the balance of the ecosystem, a considerable part of the living organisms in the lakes are destroyed due to pollution. The most important factor that 
disrupts the balance in the ecosystem is the unconscious exploitation of the environment to meet the luxurious living needs of the growing population. Studies on zooplanktonic organisms, which constitute the nutrients of many fish species in their younger periods and that transform the plant foods into animal proteins in the aquatic environment, have also been accelerated (Güher 1999).

As a result of the increase in industrial, domestic, and agricultural waste disposal into water systems, there is an accumulation of highly nutritious elements leading to eutrophication.

Since excessive phytoplankton growth and biological pollution are involved in eutrophication, plankton studies are important to provide information about ecosystems on topics such as the biodiversity of the lakes, pollution, and trophic levels. Turkey is considered as rich in terms of inland water resources. It is necessary to know the inland waters, aquatic organisms, and their distribution in our country for using these inland water resources efficiently.

No previous studies have been conducted in Demrek Dam Lake, where zooplankton species diversity and some water quality characteristics were investigated. On the other hand, this study, which is carried out in the dam lake, is important in terms of being an example for the next studies.

\section{Materials and Methods}

The study was carried out between April 2013 and March 2014 in Demrek Dam Lake, which has 48 ha lake area, in Hatay province Hassa district (Figure 1). Demrek Dam Lake has $1995 \mathrm{hm}^{3}$ water storage volume, 276 ha irrigation capacity, its construction started in 1997, its construction was completed in 2006 and it was put into operation in 2006 (Anonymous 2006).

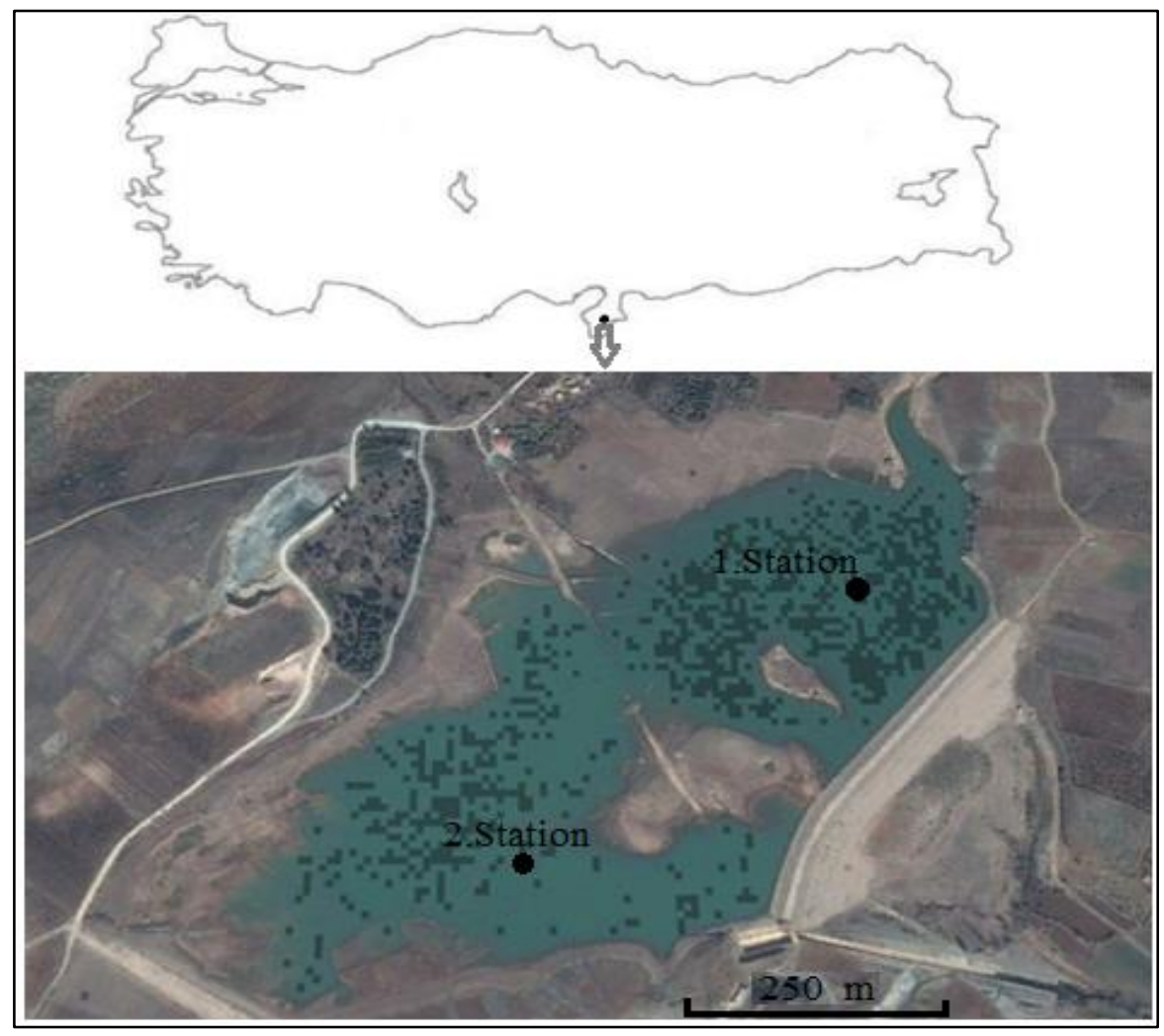

Figure 1. Demrek Dam Lake and sampling stations.

Zooplankton samples were taken from 2 stations with horizontal and vertical hauls by using $60 \mu \mathrm{m}$ mesh size plankton nets monthly for systematic analyses. Using the Nansen bottle, two liters of water samples were collected from each depth of the different water layers (surface, medium and deep) of both stations. Water quality parameters and chlorophyll- $a$ were analyzed from water samples. For the chlorophyll- $a$ analysis and chemical analyzes, one lt and $100 \mathrm{ml}$ of the total water samples were used, respectively. The remaining part (4.9 lt) was filtered from a collector having a mesh size of $60 \mu \mathrm{m}$ for zooplankton analyses. All zooplankton samples were fixed in $4 \%$ formaldehyde. Dissolved oxygen, water temperature, and conductivity were measured directly in the field using digital instruments (oxygen and temperature: YSI model 52 oxygen meter; conductivity: YSI model 30 salinometer). YSI 950 photometer and its procedure were used to determine nitrite nitrogen, nitrate nitrogen, phosphate phosphorus, Organic phosphate; the method in APHA 1995 was used to determine chlorophyll- $a$ 
spectrophotometrically. Secchi depth was measured using a Secchi disk with a diameter of $20 \mathrm{~cm}$.

The lowest depth at the stations was $4 \mathrm{~m}$ (station 1) and $7 \mathrm{~m}$ (station 2) in October, and the highest depth was $9 \mathrm{~m}$ and $12 \mathrm{~m}$ in May, respectively. Species identifications were made using a binocular microscope according to the works of Edmondson (1959), Scourfield and Harding (1966), Dussart (1967), Kiefer and Fryer (1978), Koste (1978), Negrea (1983), Segers (1995), De Smet (1996, 1997), Nogrady and Segers (2002), Hołyńska et al. (2003) and Benzie (2005). Zooplankton count was performed using an inverted microscope in a petri dish with $2 \mathrm{~mm}$ lines at the bottom. The sample cup was made homogenized by shaking and $2 \mathrm{cc}$ subsample was taken from the cup and it was placed in a petri dish and the individuals of each species were separately counted. This process has been repeated 45 times.

CTM tolerance of the species (SPSS 20.1). Duncan's multiple range test (DMRT) was carried out for post hoc mean comparisons. Regression analysis was also carried out to evaluate the relationship between acclimation temperature and CTMin and CTMax $(\mathrm{p} \leq 0.05)$.

\section{Results}

Secchi disk depth reached the maximum depth of $160 \mathrm{~cm}$ in May and the minimum depth of $55 \mathrm{~cm}$ in September, with a mean value of $100.42 \pm 38.99 \mathrm{~cm}$ (Table 1, Figure 2). The water temperature was close to regional seasonal norms, increased from spring to summer, and decreased from autumn to winter. Thus, it was ranged from $13.58^{\circ} \mathrm{C}$ in December to $31.42^{\circ} \mathrm{C}$ in August (annual average $21.06 \pm 5.96^{\circ} \mathrm{C}$ )

The highest and the lowest dissolved oxygen values were recorded in February and July as 9.70 and $6.83 \mathrm{mg} / \mathrm{l}$, respectively (average $8.33 \pm 0.99 \mathrm{mg} / \mathrm{l}$, over the study period; Table 1, Figure 2).
Nitrate and nitrite levels showed similar patterns during the study period and maximum levels were recorded in December and January $(0.595 \mathrm{mg} / \mathrm{l}$ and $0.056 \mathrm{mg} / \mathrm{l})$, respectively. The minimum nitrate level was $0.035 \mathrm{mg} / \mathrm{l}$ in November, but the minimum nitrite level was $0.009 \mathrm{mg} / \mathrm{l}$ in November and March (Figure 2). The mean nitrate and nitrite concentrations were $0.43 \pm 0.19 \mathrm{mg} / 1$ and $0.02 \pm 0.01$ $\mathrm{mg} / \mathrm{l}$ at the end of the study (Table 1).

Hardness showed irregular ups and downs in the summer, increasing properly from September to February, but remained almost stable in spring. The average, maximum, and minimum total hardness values were $172 \pm 17.27 \mathrm{mg} / \mathrm{l}, 205 \mathrm{mg} / \mathrm{l}$, and 133.33 $\mathrm{mg} / \mathrm{l}$ respectively.

The average silica level was $1.17 \pm 0.28 \mathrm{mg} / \mathrm{l}$. Silica concentrations were observed as $0.635 \mathrm{mg} / \mathrm{l}$ in May, gradually increased to $1.588 \mathrm{mg} / \mathrm{l}$ in August, decreased to $0.932 \mathrm{mg} / \mathrm{l}$ until December, and reincreased to $1.422 \mathrm{mg} / \mathrm{l}$ in May.

Phosphate, the most vital nutrient affecting the productivity of natural water resources, was $0.14 \pm 0.03 \mathrm{mg} / \mathrm{l}$, on average. The highest and the lowest phosphate values recorded in February and June were $0.192 \mathrm{mg} / \mathrm{l}$ and $0.104 \mathrm{mg} / \mathrm{l}$, respectively (Table 1, Figure 2). Phosphate levels increased during the summer until August following a gradual decrease this month. It increased from October to February and decreased from here until June. The maximum, minimum, and mean organic phosphate values were $1.83 \mathrm{mg} / \mathrm{l}$ (November at the first station), $0.38 \mathrm{mg} / \mathrm{l}$ (January at the second station), and $1.09 \pm 0.56 \mathrm{mg} / \mathrm{l}$, respectively (Table 1 , Figure 2).

Chlorophyll- $a$ ranging from $0.013 \mathrm{mg} / \mathrm{l}$ in March to $0.086 \mathrm{mg} / \mathrm{l}$ in June and September was averaged to be $0.05 \pm 0.03 \mathrm{mg} / \mathrm{l}$ (Table 1, Fig. 2). Chlorophyll a fluctuated irregularly from April to September, and it decreased from here to March.

Table 1. Maximum, minimum, and average values of water quality parameters.

\begin{tabular}{c|c|c|c|c|c|c|c|c|c|c}
\hline & $\begin{array}{c}\text { Secchi- } \\
\text { disk(cm) }\end{array}$ & $\begin{array}{c}\text { Temp } \\
\left({ }^{\circ} \mathbf{C}\right)\end{array}$ & $\begin{array}{c}\text { DO } \\
(\mathbf{m g} / \mathbf{l})\end{array}$ & $\begin{array}{c}\text { Chl-a } \\
(\mathbf{m g} / \mathbf{l})\end{array}$ & $\begin{array}{c}\mathbf{N O}_{2}-\mathbf{N} \\
(\mathbf{m g} / \mathbf{l})\end{array}$ & $\begin{array}{c}\text { NO3-N } \\
(\mathbf{m g} / \mathbf{l})\end{array}$ & $\begin{array}{c}\text { SiO-Si } \\
(\mathbf{m g} / \mathbf{l})\end{array}$ & $\begin{array}{c}\text { PO4-P } \\
(\mathbf{m g} / \mathbf{l})\end{array}$ & $\begin{array}{c}\text { Hard- } \\
\text { ness }\end{array}$ & $\begin{array}{c}\text { Org. } \\
\text { PO4 } \\
(\mathbf{m g} / \mathbf{l})\end{array}$ \\
\hline Max & 160.00 & 31.42 & 9.70 & 0.086 & 0.056 & 0.595 & 1.588 & 0.192 & 205.00 & 1.83 \\
\hline Min & 55.00 & 13.58 & 6.83 & 0.013 & 0.009 & 0.035 & 0.635 & 0.104 & 133.33 & 0.38 \\
\hline \multirow{2}{*}{ Average } & 100.42 & 21.06 & 8.33 & 0.05 & 0.02 & 0.43 & 1.17 & 0.14 & 172.36 & 1.09 \\
& \pm 38.99 & \pm 5.96 & \pm 0.99 & \pm 0.03 & \pm 0.01 & \pm 0.19 & \pm 0.28 & \pm 0.03 & \pm 17.27 & \pm 0.56 \\
\hline
\end{tabular}




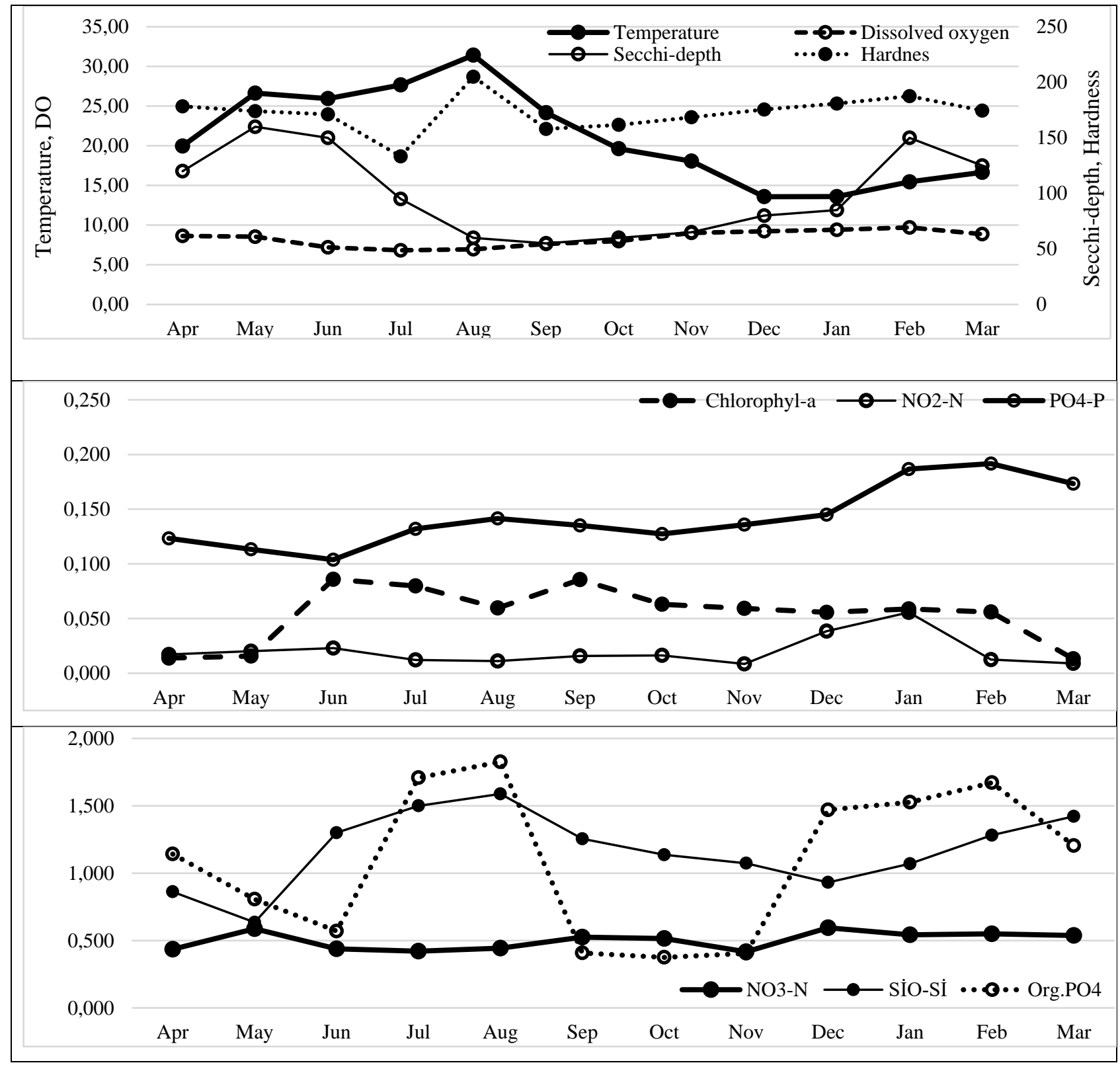

Figure 2. Monthly change of water quality parameters.

The zooplankton taxa identified in Demrek Dam Lake are shown in Table 2. The zooplankton assemblage included 63 species. Rotifera had the highest proportion with 45 taxa, followed by Cladocera with 11 species and Copepoda with 7 species.

A. priodonta, K. cochlearis, $P$. dolichoptera, $S$. stylata $B$. longirostris, $D$. birgei and $D$. rostrata were present throughout the whole study period. It was determined that these species were followed by $A$. ovalis, F. longiseta, $K$. valga, which were found for 11 months, and T. similis, which were found for 10 months.

Copepoda were found for 8 months. The least recorded species were as follows: $A$. fissa, $B$. budapestinensis, B. urceolaris, B. quadridentatus, $B$. nilsoni, C. adriatica, C. colurus, Conochiloides sp., D. epicharis, E. dilatata, H. oxyuris, K. tecta, L. closterocerca, L. luna,

L. hastata, L. hamata, L. stenroosi, L. tenuiseta, L. patella, L. rhomboides, $L$. ovalis, $L$. salpina, $P$. quadricornis, $S$. longicaudum, T. patina, T. porcellus, T. tigris, A. guttata, C. sphaericus, I. sordidus, P. laevis, C. vicinus, D. bicuspidatus, M. albidus, M. leuckarti, P. chiltoni, B. minutus, $N$. hibernica

In terms of numbers, according to the monthly distribution of the groups, the highest numbers of Rotifera were found with 22 taxa in July, followed by 21 taxa recorded in November, 20 taxa in October, but only 10 taxa were determined in April. Cladocera showed the highest number of taxa in May with 9 taxa, followed by April, June, July, October, and March with 7 taxa and November with 6 taxa. Only 3 species of Cladocera was found in January. Copepoda showed the maximum diversity with 3 taxa in July and 
October, followed by June and November with 2 taxa and April, September, January, March with 1 taxa. Copepoda species did not appear in May, August, December, and February. Total zooplankton was the highest with 32 species in July, followed by October with 30 species. It was determined to be the least with 18 species in April and August (Table 2).

Table 2. Zooplankton species list and monthly availability.

\begin{tabular}{|c|c|c|c|c|c|c|c|c|c|c|c|c|}
\hline & $\begin{array}{l}\text { Apr } \\
2013\end{array}$ & May & Jun & Jul & Aug & Sep & Oct & Nov & Dec & $\begin{array}{l}\text { Jan } \\
2014 \\
\end{array}$ & Feb & Mar \\
\hline \multicolumn{13}{|l|}{ Rotifera } \\
\hline Anuraeopsis fissa Gosse, 1851 & & & + & + & & + & & & & & & \\
\hline Ascomorpha ovalis (Bergendahl, 1892) & & + & + & + & + & + & + & + & + & + & + & + \\
\hline Asplanchna priodonta Gosse, 1850 & + & + & + & + & + & + & + & + & + & + & + & + \\
\hline Brachionus angularis Gosse, 1851 & + & + & + & + & + & + & & + & + & & & \\
\hline Brachionus budapestinensis Daday, 1885 & & & & + & & & + & + & & & & \\
\hline Brachionus urceolaris Müller, 1773 & + & & & & & & & & & & & \\
\hline Brachionus quadridentatus Hermann, 1783 & & & & + & & & + & + & & & & \\
\hline Brachionus nilsoni Ahlstrom, 1940 & & + & & & & & & & & & & \\
\hline Cephalodella gibba (Ehrenberg, 1830) & & + & + & + & & & + & + & + & + & + & + \\
\hline Collotheca pelagica (Rousselet, 1893) & & & + & + & & & & + & + & & & \\
\hline Colurella adriatica Ehrenberg, 1831 & & & & & & & & & & + & + & \\
\hline Colurella colurus (Ehrenberg, 1830) & + & & & & & & & & & & & \\
\hline Conochiloides sp. & & & & & & + & + & + & & & + & \\
\hline Dicranophorus epicharis Harring \& Myers, 1928 & + & & & & & & & & & & & \\
\hline Euchlanis dilatata Ehrenberg, 1832 & & & & & & & & & + & + & + & \\
\hline Filinia longiseta (Ehrenberg, 1834) & & + & + & + & + & + & + & + & + & + & + & + \\
\hline Filinia opoliensis (Zacharias, 1898) & & & + & + & & + & + & + & & + & & \\
\hline Hexarthra oxyuris (Sernov, 1903) & & & & & & & & & + & & & \\
\hline Itura aurita (Ehrenberg, 1830) & & & & + & + & + & + & + & & + & + & \\
\hline Keratella tecta $($ Gosse, 1851$)$ & & & + & + & & + & & & & & & \\
\hline Keratella cochlearis (Gosse, 1851) & + & + & + & + & + & + & + & + & + & + & + & + \\
\hline Keratella valga (Ehrenberg, 1834) & & + & + & + & + & + & + & + & + & + & + & + \\
\hline Lecane closterocerca (Schmarda, 1859) & & & & & & & & & & + & & + \\
\hline Lecane luna (Müller, 1776) & & & & & & & & & & & & + \\
\hline Lecane lunaris (Ehrenberg, 1832) & & & & & & & + & & + & + & & + \\
\hline Lecane hastata (Murray, 1913) & & & & & & & & + & & & & \\
\hline Lecane hamata (Stokes, 1896) & & & & & & & & & & & + & \\
\hline Lecane stenroosi (Meissner, 1908) & & & + & & & & & & & & & \\
\hline Lecane tenuiseta Harring, 1914 & & & & & & & & + & & & & \\
\hline Lepadella patella (Müller, 1773) & & + & & & & + & & & & & + & \\
\hline Lepadella rhomboides (Gosse, 1886) & & & & & & & + & & & & & \\
\hline Lepadella ovalis (Bergendahl, 1892) & + & & & & & & & & & & & \\
\hline Lophocharis salpina (Ehrenberg, 1834) & & & & & & & & & + & & & \\
\hline Notholca squamula (Müller, 1786) & & & + & + & & & & + & + & + & + & + \\
\hline Platyias quadricornis (Ehrenberg, 1832) & & & & & & & + & & & & & \\
\hline Polyarthra dolichoptera Idelson, 1925 & + & + & + & + & + & + & + & + & + & + & + & + \\
\hline Rotaria neptunia (Ehrenberg, 1830) & + & & + & + & & & + & & & & & + \\
\hline Scaridium longicaudum (Müller, 1786) & & & & & + & & & & & & & \\
\hline Sychaeta stylata Wierzejski, 1893 & + & + & + & + & + & + & + & + & + & + & + & + \\
\hline Testudinella patina (Hermann, 1783) & & & & + & + & & & & & & & \\
\hline Trichocerca similis (Wierzeski, 1893) & & + & + & + & + & + & + & + & + & & + & + \\
\hline Trichocerca pusilla (Jennings, 1903) & & + & + & + & + & + & & & & + & & \\
\hline Trichocerca porcellus (Gosse, 1851) & & & & & & & + & & & & & \\
\hline Trichocerca tigris (Müller, 1786) & & & + & & & & & + & & & & \\
\hline Trichotria tetractis (Ehrenberg, 1830) & & & & + & & & + & + & & & + & \\
\hline Total rotifer & 10 & 13 & 19 & 22 & 13 & 16 & 20 & 21 & 16 & 16 & 17 & 14 \\
\hline \multicolumn{13}{|l|}{ Cladocera } \\
\hline Bosmina longirostris (Müller, 1785) & + & + & + & + & + & + & + & + & + & + & + & + \\
\hline Ceriodaphnia pulchella Sars, 1862 & + & + & & + & & & + & & & & + & + \\
\hline Diaphanosoma birgei Korinek,1981 & + & + & + & + & + & + & + & + & + & + & + & + \\
\hline Macrothrix laticornis (Jurine, 1820) & + & + & + & + & + & & + & + & & & & \\
\hline Moina micrura Kurz, 1875 & & + & + & + & + & + & + & + & & & & \\
\hline Alona guttata Sars, 1862 & + & + & + & + & & & + & + & + & & & + \\
\hline Coronatella rectangula (Sars, 1862) & & & & & & & & & & & + & \\
\hline Chydorus sphaericus (Müller, 1776) & & + & & & & & & & & & & + \\
\hline
\end{tabular}


Table 2. Continued.

\begin{tabular}{|l|l|l|l|l|l|l|l|l|l|l|l|l|}
\hline & $\begin{array}{l}\text { Apr } \\
\mathbf{2 0 1 3}\end{array}$ & May & Jun & Jul & Aug & Sep & Oct & Nov & Dec & $\begin{array}{l}\text { Jan } \\
\mathbf{2 0 1 4}\end{array}$ & Feb & Mar \\
\hline Cladocera & & & & & & & & & & & & \\
\hline Disparalona rostrata (Koch, 1841) & + & + & + & + & + & + & + & + & + & + & + & + \\
\hline Ilyocryptus sordidus (Lievin, 1848) & + & & & & & & & & & & & + \\
\hline Pleuroxus laevis Sars, 1862 & & + & + & & & & & & & & & \\
\hline Total cladocer & $\mathbf{7}$ & $\mathbf{9}$ & $\mathbf{7}$ & $\mathbf{7}$ & $\mathbf{5}$ & $\mathbf{4}$ & $\mathbf{7}$ & $\mathbf{6}$ & $\mathbf{4}$ & $\mathbf{3}$ & $\mathbf{5}$ & $\mathbf{7}$ \\
\hline Copepoda & & & & & & & & & & & & \\
\hline Cyclops vicinus Ulyanin, 1875 & + & & & & & & + & & & & & + \\
\hline Diacyclops bicuspidatus (Claus, 1857) & & & & & & & & & & + & & \\
\hline Macrocyclops albidus (Jurine, 1820) & & & & & & & + & & & & & \\
\hline Mesocyclops leuckarti (Claus, 1857) & & & + & + & & & + & & & & & \\
\hline Paracyclops chiltoni (Thomson, 1882) & & & + & & & & & + & & & & \\
\hline Bryocamptus minutus (Claus, 1863) & & & & + & & + & & & & & & \\
\hline Nitokra hibernica (Brady, 1880) & & & & + & & & & + & & & \\
\hline Total copepod & $\mathbf{1}$ & $\mathbf{0}$ & $\mathbf{2}$ & $\mathbf{3}$ & $\mathbf{0}$ & $\mathbf{1}$ & $\mathbf{3}$ & $\mathbf{2}$ & $\mathbf{0}$ & $\mathbf{1}$ & $\mathbf{0}$ & $\mathbf{1}$ \\
\hline Total zooplankton & $\mathbf{1 8}$ & $\mathbf{2 2}$ & $\mathbf{2 8}$ & $\mathbf{3 2}$ & $\mathbf{1 8}$ & $\mathbf{2 1}$ & $\mathbf{3 0}$ & $\mathbf{2 9}$ & $\mathbf{2 0}$ & $\mathbf{2 0}$ & $\mathbf{2 2}$ & $\mathbf{2 2}$ \\
\hline
\end{tabular}

Table 3. Monthly abundance of zooplankton

\begin{tabular}{|c|c|c|c|c|c|c|}
\hline $\begin{array}{ll}\text { Species } & \text { Months } \\
\end{array}$ & April 2013 & May & June & July & Aug & Sept \\
\hline \multicolumn{7}{|l|}{ Rotifera } \\
\hline A. fissa & & & 6702 & 1126 & & 355 \\
\hline A. ovalis & & 1858 & 4204 & 74275 & 1879 & 15074 \\
\hline A. priodonta & 728 & 9299 & 13241 & 9081 & 4442 & 4402 \\
\hline B. angularis & 666 & 1423 & 909 & 15268 & 728 & 1634 \\
\hline B. budapestinensis & & & & 293 & & \\
\hline B. quadridentatus & & & & 327 & & \\
\hline C. gibba & & 306 & 303 & 502 & & \\
\hline C. pelagica & & & 758 & 248 & & \\
\hline C. colurus & 259 & & & & & \\
\hline Conochiloides sp & & & & & & 1862 \\
\hline D. epicharis & 476 & & & & & \\
\hline \multicolumn{7}{|l|}{ E. dilatata } \\
\hline F. longiseta & & 1000 & 1352 & 2519 & 571 & 939 \\
\hline F. opoliensis & & & 294 & 250 & & 692 \\
\hline \multicolumn{7}{|l|}{ H. oxyuris } \\
\hline I. aurita & & & & 2055 & 284 & 370 \\
\hline K. tecta & & & 1251 & 327 & & 357 \\
\hline K. cochlearis & 3686 & 13508 & 5849 & 9333 & 1270 & 852 \\
\hline K. valga & & 4686 & 2552 & 10566 & 2277 & 2491 \\
\hline \multicolumn{7}{|l|}{ L. closterocerca } \\
\hline \multicolumn{7}{|l|}{ L. luna } \\
\hline \multicolumn{7}{|l|}{ L. lunaris } \\
\hline \multicolumn{7}{|l|}{ L. hamata } \\
\hline L. stenroosi & & & 256 & & & \\
\hline L. patella & & 1143 & & & & 365 \\
\hline \multicolumn{7}{|l|}{ L. salpina } \\
\hline N. squamula & & & 769 & 333 & & \\
\hline \multicolumn{7}{|l|}{ P. quadricornis } \\
\hline P. dolichoptera & 18620 & 4530 & 4440 & 19608 & 4719 & 23590 \\
\hline R. neptunia & 667 & & 825 & 770 & & \\
\hline S. stylata & 11007 & 6251 & 9802 & 21244 & 712 & 14023 \\
\hline T. patina & & & & 250 & 313 & \\
\hline T. similis & & 625 & 13036 & 6355 & 822 & 1987 \\
\hline T. pusilla & & 625 & 10852 & 2766 & 15397 & 706 \\
\hline T. tigris & & & 513 & & & \\
\hline T. tetractis & & & & 251 & & \\
\hline Average rotifer & $\begin{array}{l}4514 \pm \\
6763.08 \\
\end{array}$ & $\begin{array}{l}371 \pm \\
4139.50\end{array}$ & $\begin{array}{l}4100 \pm \\
4529.23\end{array}$ & $\begin{array}{l}8079 \pm \\
16171.94\end{array}$ & \begin{tabular}{|l|}
$2785 \pm$ \\
4248.77 \\
\end{tabular} & $4356 \pm 6906.58$ \\
\hline
\end{tabular}


Table 3. Continued.

\begin{tabular}{|c|c|c|c|c|c|c|c|c|}
\hline $\begin{array}{ll}\text { Species } & \text { Months }\end{array}$ & \multicolumn{2}{|c|}{ April 2013} & May & \multicolumn{2}{|c|}{ June } & July & Aug & Sept \\
\hline \multicolumn{9}{|l|}{ Cladocera } \\
\hline B. longirostris & \multicolumn{2}{|l|}{1567} & 5983 & \multicolumn{2}{|c|}{11564} & 4372 & 33154 & 2271 \\
\hline C. pulchella & \multicolumn{2}{|l|}{15423} & 13799 & & & 1154 & & \\
\hline D. birgei & \multicolumn{2}{|l|}{1095} & 1508 & 640 & & 3787 & 28102 & 10512 \\
\hline M. laticornis & \multicolumn{2}{|l|}{1664} & 283 & 43 & 438 & & 455 & \\
\hline M. micrura & & & 287 & 28 & 1928 & & 851 & 713 \\
\hline C. rectangula & 1997 & & 6990 & 46 & 894 & & & \\
\hline A. guttata & & & & & & & & \\
\hline D. rostrata & 913 & & 2087 & 10 & 1269 & & 690 & 763 \\
\hline Average cladocer & \begin{tabular}{|l|}
$3777 \pm$ \\
5719.13
\end{tabular} & & $\begin{array}{l}4420 \pm \\
4918.21\end{array}$ & & $\begin{array}{l}0 \pm \\
3.06\end{array}$ & & $\begin{array}{l}12650 \pm \\
16508.74\end{array}$ & $3565 \pm 4687.58$ \\
\hline Copepoda & & & & & & & & \\
\hline C. vicinus & 3788 & & & & & & & \\
\hline D. bicuspidatus & & & & & & & & \\
\hline M. albidus & & & & & & & & \\
\hline M. leuckarti & & & & 30 & 580 & & & \\
\hline P. chiltoni & & & & 30 & & & & \\
\hline N. hibernica & & & & & 763 & & & \\
\hline Average copepod & $3788 \pm 0$ & & $\mathbf{0} \pm \mathbf{0}$ & & $672 \pm$ & $\mathbf{1 2 9 , 4 0}$ & $\mathbf{0} \pm \mathbf{0}$ & $\mathbf{0} \pm \mathbf{0}$ \\
\hline Average zooplankton & \begin{tabular}{|l|}
$4026 \pm$ \\
422.30
\end{tabular} & & $\begin{array}{l}4095 \pm \\
2386.59\end{array}$ & & \begin{tabular}{l|l}
$1 \pm$ & 3576 \\
2.51 & 3954 \\
\end{tabular} & \pm .25 & $\begin{array}{l}7717 \pm \\
6647.33\end{array}$ & $\begin{array}{l}3960 \pm \\
2320.57\end{array}$ \\
\hline Species Months & Oct & Nov & \begin{tabular}{l|l} 
Dec \\
\end{tabular} & & Jan 2014 & Febr & Marc & Average \\
\hline Rotifera & & & & & & & & \\
\hline A. fissa & & & & & & & & $2728 \pm 3463.39$ \\
\hline A. ovalis & 2616 & 889 & 290 & & 784 & 4371 & & $10624 \pm 22772.80$ \\
\hline A. priodonta & 27286 & 1080 & 20476 & & 1612 & 1201 & 891 & $7812 \pm 8641.56$ \\
\hline B. angularis & & 521 & 276 & & & & & $2678 \pm 5107.07$ \\
\hline B. budapestinensis & 2483 & 3141 & & & & & & $1972 \pm 1491.09$ \\
\hline B. quadridentatus & & & & & & & & $327 \pm 0$ \\
\hline C. $g i b b a$ & 451 & 520 & 295 & & 1863 & 692 & & $617 \pm 521.99$ \\
\hline C. pelagica & & 272 & 279 & & & & & $389 \pm 246.19$ \\
\hline C. colurus & & & & & & & & $259 \pm 0$ \\
\hline Conochiloides sp & 1403 & 3867 & & & & 939 & & $2018 \pm 1289.13$ \\
\hline D. epicharis & & & & & & & & $476 \pm 0$ \\
\hline E. dilatata & & & 267 & & 882 & 293 & & $481 \pm 347.81$ \\
\hline F. longiseta & 14606 & 2194 & 537 & & 907 & 999 & 253 & $2352 \pm 4121.02$ \\
\hline F. opoliensis & 1462 & 771 & & & 293 & & & $627 \pm 466.21$ \\
\hline H. oxyuris & & & 273 & & & & & $273 \pm 0$ \\
\hline I. aurita & 12132 & 257 & & & 295 & 2056 & & $2493 \pm 4330.34$ \\
\hline K. tecta & & & & & & & & $645 \pm 525.03$ \\
\hline K. cochlearis & 48237 & 6669 & 2949 & & 38695 & 10508 & 640 & $11850 \pm 15441.51$ \\
\hline K. valga & 76334 & 13275 & 2008 & & 1858 & 1567 & & $11761 \pm 23048.84$ \\
\hline L. closterocerca & & & & & 291 & & 2562 & $1427 \pm 1605.84$ \\
\hline L. luna & & & & & & & 258 & $258 \pm 0$ \\
\hline L. lunaris & 998 & & 276 & & 577 & & 945 & $699 \pm 338.49$ \\
\hline L. hamata & & & & & & 287 & & $287 \pm 0$ \\
\hline L. stenroosi & & & & & & & & $256 \pm 0$ \\
\hline L. patella & & & & & & 295 & & $601 \pm 470.69$ \\
\hline L. salpina & & & 280 & & & & & $280 \pm 0$ \\
\hline N. squamula & & 274 & 4845 & & 2944 & 557 & 262 & $1426 \pm 1782.20$ \\
\hline P. quadricornis & 325 & & & & & & & $325 \pm 0$ \\
\hline P. dolichoptera & 27206 & 25262 & 1566 & & 26716 & 12435 & 15574 & $15356 \pm 9593.48$ \\
\hline R. neptunia & 332 & & & & & & 927 & $704 \pm 228.30$ \\
\hline S. stylata & 204367 & 4407 & 16644 & & 13529 & 8485 & 1936 & $26034 \pm 56482.24$ \\
\hline T. patina & & & & & & & & $282 \pm 44.55$ \\
\hline T. similis & 2714 & 8982 & 172 & & & 1079 & 989 & $3676 \pm 4351.43$ \\
\hline
\end{tabular}


Table 3. Continued.

\begin{tabular}{|c|c|c|c|c|c|c|c|}
\hline Species $\quad$ Months & Oct & Nov & Dec & Jan 2014 & Febr & Marc & Average \\
\hline T. pusilla & & & & 285 & & & $389 \pm 6435.50$ \\
\hline T. tigris & & 265 & & & & & $389 \pm 175.36$ \\
\hline T. tetractis & 318 & 276 & & & 317 & & $291 \pm 32.81$ \\
\hline Average rotifer & \begin{tabular}{|l|}
$24898 \pm$ \\
50776.53
\end{tabular} & \begin{tabular}{|l|}
$4051 \pm$ \\
6401.44
\end{tabular} & $\begin{array}{l}\text { 3215士 } \\
6167.40\end{array}$ & $\begin{array}{l}6102 \pm \\
11515.92\end{array}$ & $\begin{array}{l}2880 \pm \\
3964.39\end{array}$ & $\begin{array}{l}2294 \pm \\
4462.00\end{array}$ & $5920 \pm 6180,93$ \\
\hline \multicolumn{8}{|l|}{ Cladocera } \\
\hline B. longirostris & 14780 & 1653 & 2235 & 3583 & 803 & 2188 & $7013 \pm 9291.93$ \\
\hline C. pulchella & 1988 & & & & 276 & 9610 & $7042 \pm 6759.93$ \\
\hline D. birgei & 2719 & 3755 & 3210 & 2209 & 2125 & 1210 & $5073 \pm 947.53$ \\
\hline M. laticornis & 2856 & 772 & & & & & $986 \pm 947.53$ \\
\hline M. micrura & 2844 & 255 & & & & & $1023 \pm 995.35$ \\
\hline C. rectangula & 935 & 268 & 315 & & & 375 & $1530 \pm 2278.08$ \\
\hline A. guttata & & & & & 262 & & $262 \pm 0$ \\
\hline D. rostrata & 4726 & 1291 & 1054 & 946 & 577 & 386 & $1315 \pm 1158.28$ \\
\hline Average cladocer & \begin{tabular}{|l}
$4407 \pm$ \\
4713.55 \\
\end{tabular} & $\begin{array}{l}1332 \pm \\
1310.06\end{array}$ & $\begin{array}{l}1704 \pm \\
1278.26\end{array}$ & \begin{tabular}{|l|}
$2246 \pm$ \\
1318.89 \\
\end{tabular} & $\begin{array}{l}809 \pm \\
769.58\end{array}$ & $\begin{array}{l}2754 \pm \\
3904.36 \\
\end{array}$ & $3504 \pm 3108.37$ \\
\hline \multicolumn{8}{|l|}{ Copepoda } \\
\hline C. vicinus & 750 & & & & & 3120 & $2553 \pm 1596.48$ \\
\hline D. bicuspidatus & & & & 288 & & & $288 \pm 0$ \\
\hline M. albidus & 993 & & & & & & $993 \pm 0$ \\
\hline M. leuckarti & 987 & & & & & & $623 \pm 344.053$ \\
\hline P. chiltoni & & 266 & & & & & $285 \pm 26.16$ \\
\hline N. hibernica & & 780 & & & & & $772 \pm 12.02$ \\
\hline Average copepod & \begin{tabular}{|l|}
$523 \pm$ \\
138,60
\end{tabular} & \begin{tabular}{|l|}
$523 \pm$ \\
363,45
\end{tabular} & $\mathbf{0} \pm \mathbf{0}$ & $288 \pm 0$ & $\mathbf{0} \pm \mathbf{0}$ & $3120 \pm 0$ & $768 \pm 1285.49$ \\
\hline Average zooplankton & $\begin{array}{l}9943 \pm \\
13096.48\end{array}$ & \begin{tabular}{|l|}
$1969 \pm$ \\
1848.16
\end{tabular} & $\begin{array}{l}2459 \pm \\
1608.47\end{array}$ & $\begin{array}{l}2879 \pm \\
2958.18\end{array}$ & $\begin{array}{l}1844 \pm \\
1485.37\end{array}$ & $\begin{array}{l}2723 \pm \\
413.89\end{array}$ & $3398 \pm 2319.00$ \\
\hline
\end{tabular}

The most abundant species from Rotifera was $S$. stylata (annual average $26034 \pm 56482.24$ ind.$/ \mathrm{m}^{3}$ ). $P$. dolichoptera $\left(15356 \pm 9593.48\right.$ ind. $\left./ \mathrm{m}^{3}\right)$ and $K$. cochlearis $\left(11850 \pm 15441.51 \mathrm{ind} . / \mathrm{m}^{3}\right)$ were found the second and third abundant species, respectively. The least abundant species was L. stenroosi $(256 \pm 0$ ind. $/ \mathrm{m}^{3}$ ).

In this study, the most common species belonging to Cladocera was $C$. pulchella (7042 \pm 6759.93 ind. $/ \mathrm{m}^{3}$ ) according to their annual averages. The second abundant species was $B$. longirostris $\left(7013 \pm 9291.93\right.$ ind. $\left./ \mathrm{m}^{3}\right)$ and followed by $D$. birgei $\left(5073 \pm 7700.78\right.$ ind. $\left./ \mathrm{m}^{3}\right)$. The most abundant copepod species was $C$. vicinus $(2553 \pm 1596.48$ ind. $/ \mathrm{m}^{3}$ ) followed by $M$. albidus $\left(993 \pm 0\right.$ ind. $\left./ \mathrm{m}^{3}\right)$ and $N$. hibernica $\left(772 \pm 12.02\right.$ ind. $\left./ \mathrm{m}^{3}\right)$. The least abundant cladoceran species were A. guttata $(262 \pm 0$ ind. $\left./ \mathrm{m}^{3}\right)$ and copepod $P$. chiltoni $(285 \pm 26.16$ ind. $\left./ \mathrm{m}^{3}\right)$.

Considering their monthly abundance, the most abundant species was rotifer S. stylata (October 2013, 204367 ind./ $\mathrm{m}^{3}$ ) and followed by $K$. valga (October 2013, 76334 ind./ $\mathrm{m}^{3}$ ), and A. ovalis (July $\left.2013,74275 \mathrm{ind} . / \mathrm{m}^{3}\right)$. The least abundant species was T. similis obtained in December 2013 (172 ind. $/ \mathrm{m}^{3}$ ) (Table 3).
The most abundant species were copepod $C$. vicinus (April 2013, 3788 ind. $/ \mathrm{m}^{3}$ ) and cladoceran B. longirostris (August 2013, 33154 ind. $\mathrm{m}^{3}$ ). Other abundant species were cladoceran $D$. birgei (August 2013, 28102 ind. $/ \mathrm{m}^{3}$ ), C. pulchella (April 2013, 15423 ind. $/ \mathrm{m}^{3}$ ), copepod M. albidus (October 2013, 993 ind. $\left./ \mathrm{m}^{3}\right)$, M. leuckarti (October 2013, 987 ind. $/ \mathrm{m}^{3}$ ) and $N$. hibernica $\left(780\right.$ ind. $\left./ \mathrm{m}^{3}\right)$. The least abundant species from Cladocera were A. guttata (February 2014, 262 ind. $/ \mathrm{m}^{3}$ ) and copepod P. chiltoni (November 2013, 266 ind. $/ \mathrm{m}^{3}$ ) (Table 3).

The most dominant rotifer $(24898 \pm 50776.53$ ind. $\left./ \mathrm{m}^{3}\right)$, and total zooplankton $(9943 \pm 12231.09$ ind. $/ \mathrm{m}^{3}$ ) were obtained in October 2013, cladocer in August $2013\left(12656 \pm 16508,74\right.$ ind.$\left./ \mathrm{m}^{3}\right)$ and copepod in April 2013 (3788 \pm 0 ind. $\left./ \mathrm{m}^{3}\right)$. The mean rotifer, cladocer and copepod abundance were $5920 \pm 6180.91$ ind. $/ \mathrm{m}^{3}, 3504 \pm 3108.29$ ind. $/ \mathrm{m}^{3}$ and $768 \pm 1285.49$ ind. $/ \mathrm{m}^{3}$ in the dam lake respectively. The mean zooplankton was the most abundant in October $(9943 \pm 41292.83)$, followed by August (7717 \pm 10100.33$)$ and May (4095 \pm 4317.16$)$, but the least abundant zooplankton was in February $(1844 \pm 3566.94)$ (Table 3). 
It has been determined that Rotifera, Cladocera, Copepoda and average zooplankton showed monthly irregular and unstable fluctuations, and peaked in the middle of summer and autumn. Copepod was not found in quantitative samples in May, August, September, December, and February (Figure 3, Table 3).

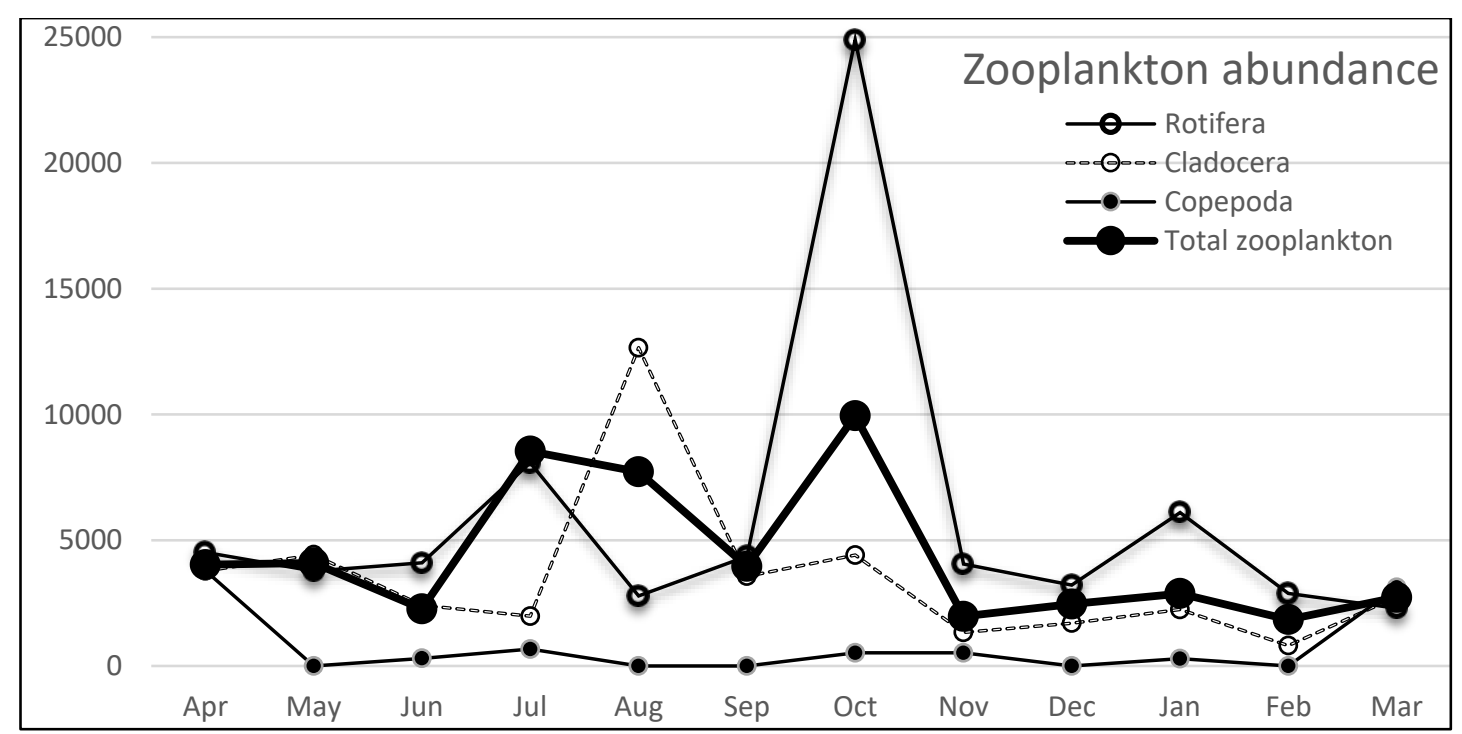

Figure 3. Monthly abundances of zooplankton.

Table 4. Relationship levels between water quality parameters and zooplankton abundance.

\begin{tabular}{|c|c|c|c|}
\hline & Rotifera & Cladocera & Zooplankton \\
\hline $\operatorname{Temp}\left({ }^{\circ} \mathrm{C}\right)$ & $\mathrm{R}^{2}=0.09$ & $\mathrm{R}^{2}=0.61$ & $\mathrm{R}^{2}=0.34$ \\
\hline DO (mg/l) & $\mathrm{R}^{2}=0.17$ & $\mathrm{R}^{2}=0.34$ & $\mathrm{R}^{2}=0.45$ \\
\hline Chl-a (mg/l) & $\mathrm{R}^{2}=0.14$ & $\mathrm{R}^{2}=0.04$ & $\mathrm{R}^{2}=0.04$ \\
\hline $\mathrm{NO}_{2}-\mathrm{N}(\mathrm{mg} / \mathrm{l})$ & $\mathrm{R}^{2}=0.01$ & $\mathrm{R}^{2}=0.05$ & $\mathrm{R}^{2}=0.08$ \\
\hline $\mathrm{NO}_{3}-\mathrm{N}$ (mg/l) & $\mathrm{R}^{2}=0.07$ & $\mathrm{R}^{2}=0.07$ & $\mathrm{R}^{2}=0.12$ \\
\hline SiO-Si (mg/l) & $\mathrm{R}^{2}=0.04$ & $\mathrm{R}^{2}=0.46$ & $\mathrm{R}^{2}=0.21$ \\
\hline $\mathrm{PO}_{4}-\mathrm{P}(\mathrm{mg} / \mathrm{l})$ & $\mathrm{R}^{2}=0.08$ & $\mathrm{R}^{2}=0.17$ & $\mathrm{R}^{2}=0.24$ \\
\hline Hardnes & $\mathrm{R}^{2}=0.54$ & $\mathrm{R}^{2}=0.57$ & $\mathrm{R}^{2}=0.43$ \\
\hline $\mathrm{Org} \mathrm{PO}_{4}(\mathrm{mg} / \mathrm{l})$ & $\mathrm{R}^{2}=0.15$ & $\mathrm{R}^{2}=0.18$ & $\mathrm{R}^{2}=0.26$ \\
\hline
\end{tabular}

A significant functional relationship was found between zooplankton and water quality parameters (hardness-rotifer, $\mathrm{R}^{2}=0.54$; temperature-cladocer, $\mathrm{R}^{2}=0.61$; hardness-cladocer, $\mathrm{R}^{2}=0.57$ ). A weak correlation was found between zooplankton and other water quality parameters (Table 4).

\section{Discussion}

Water quality parameters and zooplankton communities together form a comprehensive ecosystem having interaction between both zooplankton and phytoplankton and the water quality parameters. These interactions are directly or indirectly subjected to the complex influences, some of which results in quantitative changes (Welch 1952). Water quality parameters in the study were observed to be within the normal values for animals in the water. According to this, water temperature values $\left(13.58-31.42{ }^{\circ} \mathrm{C}\right)$ in the study generally reflect the climatic conditions of the region and they are ideal for zooplankton and their development. Mean dissolved oxygen concentrations were above $5 \mathrm{mg} / \mathrm{l}$ (6.83-9.70 mg/L) which was enough to support aquatic life, especially the zooplankton community (Karpowicz and EjsmontKarabin 2017).

The mean value of chlorophyll- $a$ was relatively high (0.013-0.086 mg /L) and indicated that the lake has a eutrophic character, according to Wetzel (1975).

Inorganic forms of nitrogen $\left(\mathrm{NO}_{3}{ }^{-}\right.$and $\left.\mathrm{NO}_{2}{ }^{-}\right)$can be used by aquatic plants and algae (Tepe and Boyd 2002). If these inorganic forms of nitrogen exceed 0.3 $\mathrm{mg} / \mathrm{l}$ (as $\mathrm{N}$ ) in spring, it means there is enough nitrogen to support summer algal blooms. The concentrations of nitrogen forms in Demrek Dam 
Lake were enough to support algae blooms and indirectly zooplankton biomass. The quality of reservoir waters generally varied between clean water and much-polluted water throughout the year in terms of nitrite values (YSKY 2012). As the nitrate-nitrogen values determined in the study were below $10 \mathrm{mg} / \mathrm{l}$, thus the reservoir waters were in the category of clean and less polluted water.

Orthophosphate values changed between 0.104 $\mathrm{mg} / \mathrm{l}$ and $0.192 \mathrm{mg} / \mathrm{l}$ and the reservoir waters generally have the second-class polluted water and the third-class polluted water in terms of phosphate according to the YSKY (2012). As a result, according to the Regulation on Surface Water Quality, reservoir water was first-class water in point of $\mathrm{NO}_{3}-\mathrm{N}$, partly also dissolved oxygen, and third class water in point of $\mathrm{NO}_{2}-\mathrm{N}$ (YSKY 2012).

The annual mean values of total phosphorus and chlorophyll-a with $0.14 \mathrm{mg} / \mathrm{L}$ and $0.05 \mathrm{mg} / \mathrm{L}$ respectively also make the lake in hyper-eutrophic class according to YSKY (2012). The Dam Lake was determined to be eutrophic in terms of average Secchi disc depth and mesotrophic in terms of nitrogen (YSKY 2012).

Since there is a close relation between phytoplankton and zooplankton because of the food chain, increases were observed in zooplankton biomass following the phytoplankton bloom. The highest amount of Rotifera was reported in the area where phytoplankton bloom occurred, as they consequently found abundant food sources (RuttnerKolisko 1974; Horn and Goldman 1994; Noges 1997). Similar results were found in the present study. In May, chlorophyll increased due to decreased zooplankton. In the following months, the amount of zooplankton was decreased with a decrease in chlorophyll- $a$ but increased with an increase in chlorophyll- $a$.

Zooplankton species diversity and abundance of Demrek Dam Lake seem to be considerably rich compared with other studies carried out in different Turkish lakes [17 Rotifera species in Yamansaz Lake (Yalım 2006); 16 Rotifera species in Hazar Lake (Tellioğlu and Şen 2002); a total of 17 species, 10 belong to Rotifera, 5 to Cladocera and 2 to Copepoda, in Lake Burdur (Altındağ and Yiğit 2002). Yıldız et al. (2007) declared that 41 species were found in Lake Marmara, including 29 Rotifera, 8 Cladocera, and 4 Copepoda. Bekleyen and Taş (2008) had found 10 species from Cladocera, 3 from Copepoda, and 18 from Rotifera (a total 31 species) in Çernek Lake. The same situation was observed in dam lakes. Results of some studies were as follow: 54 species were declared in Aslantaş Dam Lake, including 35 Rotifera, 14 Cladocera, and 5 Copepoda (Bozkurt et al. 2009; Bozkurt and Göksu 2010); totally 39 taxa declared, containing 21 rotifer, 11 cladocer and 7 copepod in Birecik Dam Lake (Bozkurt and Sağat 2008). Some others; 11 rotifer, 7 cladocer and 1 copepod, 19 species in total in Çamlıgöze Dam Lake (Dirican and Musul 2008); 12 cladocer, 5 copepod, 17 species in total in Devegeçidi Dam Lake (Bekleyen 2006); 8 cladocer, 2 copepod, 10 species in total in Çatalan Dam Lake (Aladağ et al. 2006); 18 rotifer, 9 cladocer and 4 copepod, 31 species in total in Hirfanlı Dam Lake (Yiğit and Altındağ 2005); 11 rotifer in Kesikköprü Dam Lake (Yiğit 2002); 21 rotifer, 7 cladocer and 4 copepod, 32 species in total in Kurtboğazı and Çamlıdere Dam Lakes (Demir 2005); 8 cladocer and 4 copepod, 12 species in total in İkizcetepeler Dam Lake (Alper et al. 2007); 34 rotifer at Devegeçidi Dam Lake (Bekleyen 2001) and 28 rotifer, 16 cladocer and 3 copepod, 47 species in total were declared (Bekleyen 2003).

The simultaneous presence of several species of the genus Brachionus is a good indication for the eutrophic nature of an aquatic ecosystem (Angeli 1976; Mageed 2008; Uzma 2009). Patalas (1972) noticed that in the lakes of EUA the cyclopoid copepods were more abundant in eutrophic waters than calanoid copepods.

The results of the study are in accordance with the above information and a large number of species reported by various researchers as eutrification indicators have also been identified in the study. These species are A. fissa, $N$. squamula, $P$. quadricornis, A. priodonta, $K$. cochlearis, $P$. dolichoptera, $B$. angularis, $R$. neptunia, $B$. urceolaris, B. quadridentatus, L. luna, L. lunaris, $L$. patella, T. patina, B. nilsoni, T. pusilla, T. tetractis, $E$. dilatata, $F$. longiseta, $F$. opoliensis, I. aurita, B. longirostris, M. micrura, K. tecta, D. birgei, $C$. rectangula, C. sphaericus, C. vicinus, M. leuckarti (Pourriot 1964; Hutchinson 1967; Flössner 1972; Ruttner-Kolisko 1974; Koste 1978; Braioni and Gemlini 1983; Gulati 1983; Margaritora 1985; Koste and Shiel 1986; Saksena 1987; Pejler and Bērzin̄š 1994; Smith 2001; Lucinda et al. 2004; Baião and Boavida 2005). Considering the water quality parameters and the determined species, it can be said that the Demrek Dam Lake is eutrophic. The remaining species in the study are widely distributed in the inland water of Turkey and have been identified in many studies (Ustaoğlu et al. 2012; Ustaoğlu 2015).

\section{Acknowledgements}

The authors would like to thank Ece Kılıç from Iskenderun Technical University for providing comments that improved the quality of the manuscript and for correcting the English. 


\section{References}

Aladağ AT, Erdem C, Karaytuğ S. 2006. Cladocera ve Copepoda (Crustacea) fauna of Çatalan Dam Lake (Adana, Turkey). EgeJFAS. 23(3-4): 427-428.

Alper A, Çelebi E, Çam H, Karaytuğ S. 2007. Cladocera and Copepoda (Crustacea) fauna of İkizcetepeler Dam Lake (Balıkesir, Turkey). Turk J Fish Aquat Sc. 7:71-73.

Altındağ A, Yiğit S. 2002. The zooplankton fauna of Lake Burdur. Ege JFAS. 19(1-2):129-132.

Angeli N. 1976. Influence de la pollution sur les éléments du plancton. In Pesson P, editör. La Pollution des eaux continentales. Paris: Gauthier-Villars. p. 97-134.

Anonymous 2006. General Directorate of State Hydraulic Works. [Erişim tarihi: $20 \mathrm{Haz}$ 2020]. Erișim Adresi: http://bolge06.dsi.gov.tr/isletmedekitesisler/barajg\%C3\%B6let/

APHA 1995. Standart methods for the examination of water and waste water, 19th Ed. Washington, DC: American Public Health Association 1325 p.

Baião C, Boavida MJ. 2005. Rotifers of Portuguese reservoirs in river Tejo catchment: Relations with trophic state. Limnetica 24(1-2):103-114. doi: 10.23818/limn.24.10

Bekleyen A. 2001. A Taxonomical Study on the Rotifera fauna of Devegeçidi Dam Lake (Diyarbakır-Turkey). Turk J Zool. 25(3):251-255.

Bekleyen A. 2003. A Taxonomical study on the zooplankton of Göksu Dam Lake (Diyarbakır). Turk J of Zool. 27(2):95-100.

Bekleyen A. 2006. Devegeçidi Baraj Gölü’nün (Diyarbakır) Cladocera ve Copepoda (Crustacea) faunasi. Turkey). Ege JFAS. 23(3-4):413-415.

Bekleyen A, Taş B. 2008. Zooplankton fauna of Çernek Lake. Ekoloji. 17(67):24-30.

Benzie JAH. 2005. Cladocera: the genus Daphnia (including Daphniopsis). Leiden, The Netherlands: Backhuys Publishers $376 \mathrm{p}$.

Bērzinš B, Pejler B. 1987. Rotifer occurrence in relation to pH. Hydrobiologia. 147:107-116. doi: 10.1007/bf00025733

Bozkurt A, Sagat Y. 2008. Birecik Baraj Gölü zooplaktonunun vertikal dağılımı. Journal of FisheriesSciences.com. 2(3):332-342. [in Turkish]. doi: 10.3153/jfscom.mug.200721

Bozkurt A, Göksu MZL, Altun A. 2009. Cladocera and copepoda fauna of Aslantaş Dam Lake (OsmaniyeTurkey). Journal of FisheriesSciences.com. 3(4):285-297. doi: 10.3153/jfscom.2009033

Bozkurt A, Göksu MZL. 2010. Composition and vertical distribution of Rotifera in Aslantas Dam Lake (Osmaniye-Turkey). Journal of Fisheries Sciences.com. 4(1):38-49. doi: $10.3153 /$ jscom.2010005a

Braioni MG, Gelmini D. 1983. Guide per il reconoscimento delle specie animali delle acque interne Italiane: Rotiferi monogononti. Italy: Consiglio Nazionalie delle Ricerche 181p.
Demir N. 2005. Zooplankton of two drinking water reservoirs in Central Anatolia: composition and seasonal cycle. Turk J Zool. 29:9-16.

De Smet WH. 1996. Rotifera 4: The Proalidae (Monogononta). In Dumont HJ, Nogrady T, editors. Guides to the Identification of the Microinvertebrates of the Continental Waters of the World 9. The Netherlands: SPB Academic Publishing 102 p.

De Smet WH. 1997. Rotifera 5: the Dicranophoridae. Guides to the Identification of the Microinvertebrates of the Continental Waters of the World, vol. 12. The Hague, the Netherlands: SPB Academic Publishing BV. 1-325 p.

Dirican S, Musul H. 2008. Çamlıgöze Baraj Gölü zooplankton faunası üzerine bir çalışma. Sakarya Üniv Fen Bil Derg. 12(1):17-21.

Dussart B. 1967. Les Copepodes des eaux continentales d'Europe occidentale. Tome I: Calanoides at Harpacticoides. Paris: N. Boublée \& Cie 500 p.

Edmondson WT. 1959. Rotifera. In: Edmondson WT, editör. Fresh-water Biology. NY: John Wiley \& Sons, Inc. p. 420-494.

Flössner D. 1972. Krebstiere, Crustacea. Kiemen und Blattfüsser, Branchiopoda, Fishlause, Branchiura, Tierwelt Deutschlands, 60. Teil. Jena: Veb Gustav Fischer Verlag 501 p.

Gulati RD. 1983. Zooplankton and its grazing as indicators of trophic status in Dutch lakes. Environ Monit Assess. 3:343-353. doi: $10.1007 / \mathrm{BF} 00396229$

Güher H. 1999. Mert, Erikli, Hamam ve Pedina gölleri’nin (İğneada/Kırklareli) Cladocera ve Copepoda (Crustacea) türleri üzerinde taksonomik bir çalışma. Turk J Zool. 23 (Ek sayı 1):47-53.

Hecky RE, Kilham P. 1973. Diatoms in alkaline, saline lakes: Ecology and geochemical implications. Limnol Oceanogr. 18(1):53-71. doi: 10.4319/lo.1973.18.1.0053

Hołyńska M, Reid JW, Ueda H. 2003. Genus Mesocyclops Sars, 1914. In: Ueda H, Reid JW, editors. Copepoda: Cyclopoida Genera Mesocyclops and Thermocyclops Guides to the Identification of the Microinvertebrates of the Continental Waters of the World, Volume 20. Amsterdam: Backhuys Publishers 12-214 p.

Horn AJ, Goldman CR. 1994. Limnology. New York: McGraw-Hill, inc. 576 p.

Hutchinson GE. 1967. A Treatise on Limnology. Vol. 2. Introduction to lake biology and the limnoplankton. New York: Wiley Intersci. Publ. 1115 p.

Karpowicz M, Ejsmont-Karabin J. 2017. Effect of metalimnetic gradient on phytoplankton and zooplankton (Rotifera, Crustacea) communities in different trophic conditions. Environ Monit Assess. 189(8):354-367. doi: $10.1007 / \mathrm{s} 10661-017-6055-7$

Kiefer F, Fryer G. 1978. Das Zooplankton der Binnengewasser 2. Teil. Stuttgart: E. Schweizerbart'sche Verlagsbuchhandlung $380 \mathrm{p}$.

Koste W. 1978. Rotatoria. Die radertiere mittel-europas, 2nd ed. Berlin and Stuttgart: Gebruder Borntraeger $673 \mathrm{p}$. 
Koste W, Shiel RJ. 1986. Rotifera from Australian inland waters. I. Bdelloidea (Rotifera: Digononta), II. Epiphanidae \& Brachionidae (Rotifera: Monogononta). Aust J Mar Fresh Res. 37(6):765-792.

Lucinda I, Moreno IH, Melão MGG, Matsumura-Tundisi T. 2004. Rotifers in freshwater habitats in the upper Tietê River Basin, São Paulo State, Brazil. Acta Limnol Bras. 16(3):203-224.

Mageed A. 2008. Distribution and long-term historical changes of zooplankton assemblages in Lake Manzala (South Mediterranean Sea, Egypt). Egypt J Aquat Res. 33(1):183-192.

Margaritora FG. 1985. Fauna D'Italia. Cladocera. Bologna: Calderini 399 p.

Mikschi E. 1989. Rotifer distributions in relation to temperature and oxygen content. Hydrobiologia 186187:209-214.

Negrea S. 1983. Fauna Republicii Socialiste Romania. Crustacea Volum IV. Facicula 12 Cladocera. Bucuresti: Academia Repubblici Socialiste Romania $399 \mathrm{p}$.

Noges T. 1997. Zooplankton-phytoplankton interactions in Lakes Vortsjarv, Peipsi (Estonia) and Yaskhan (Turkmenia). Hydrobiologia, Belgium, 342/343:175184.

Nogrady T, Segers H. 2002. Rotifera 6; The Asplanchnidae, Gastropodidae, Lindiidae, Microcodinidae, Synchaetidae, Trochosphaeridae. In Dumont, HJ, editor. Guides to the Identification of the Microinvertebrates of the Continental Waters of the World 18. Dordrecht, The Netherlands: Backhuys Publishers BV 264 p.

Patalas K. 1972. Crustacean plankton and the eutrophication of St. Lawrence Great Lakes. Can J Fis Aquat Sci. 29: 1451-1462. doi: 10.1139/f72-224

Pejler B, Bērziňš B. 1994. On the ecology of Lecane (Rotifera). Hydrobiologia 273:77-80. doi: 10.1007/BF00006849

Pourriot R. 1964. Etude expérimentale de variations morphologiques chez certaines espèces de Rotifères. B Soc Zool Fr. 89:555-561.

Ruttner-Kolisko A. 1974. Plankton rotifers. Biology and taxonomy. English translation of Die Binnengewiisser v. 26, part 1. Stuttgart: Schweizerbart 146 p.

Saksena ND. 1987. Rotifers as indicator of water quality, Hydrobiology, 15(5):481-485. doi: 10.1002/aheh.19870150507

Scourfield DJ, Harding JP. 1966. A key to British species of freshwater Cladocera with notes on their ecology. 3rd ed. Sciencific Publication
5. Ambleside, UK: Freshwater Biological Associasion $55 \mathrm{p}$.

Segers H. 1995. Rotifera: the Lecanidae (Monogononta). In: Dumont HJ, Nogrady T, editors. Guides to the identification of the microinvertebrates of the continental waters of the world, vol. 2. Amsterdam:

SPB Academic $226 \mathrm{p}$.

Smith DG. 2001. Pennak's freshwater invertabrates of the United States: Porifera to Crustacea, 4th edn. New York: John Wiley \& Sons 664 p.

Tellioğlu A, Şen D. 2002. A Taxonomical Study on the Rotifer Fauna of Hazar Lake (Elazığ) (in Turkish). EgeJFAS 19(1-2):205-207.

Tepe Y, Boyd CE. 2002. Nitrogen fertilization of Goldenshiner ponds. N Am J Aquacult. 64(4):284289.

doi:10.1577/1548454(2002)064<0284:NFOGSP>2.0. $\mathrm{CO} ; 2$

Ustaoğlu MR, Altındağ A, Kaya M, Akbulut N, Bozkurt A, Özdemir Mis D, Atasagun S, Erdoğan S, Bekleyen A, Saler S, Okgerman HC. 2012. A checklist of Turkish Rotifers. Turk J Zool. 36(5):607-622. doi: 10.3906/zoo-1110-1

Ustaoğlu MR. 2015. An Updated Zooplankton Biodiversity of Turkish Inland Waters. LimnoFish. 1(3):151-159. doi: 10.17216/LimnoFish-5000151941

Uzma A. 2009. Studies on plankton communities of some eutrophic water bodies of Aligarh [M.Sc. Thesis].Aligarh Muslim University. $145 \mathrm{p}$.

Welch PS. 1952. Limnology, 2nd ed. New York: Mc Graw-Hill Book Company 538 p.

Wetzel GR. 1975. Limnology. Philadelphia: W.B. Sauders Company $743 \mathrm{p}$.

Yalım FB. 2006. Rotifera fauna of Yamansaz Lake (Antalya) in South-West of Turkey. EgeJFAS. 23(34):395-397.

Yiğit S. 2002. Seasonal fluctiation in the Rotifer Fauna of Kesikköprü Dam Lake (Ankara, Turkey). Turk J Zool. 26(4):341-348.

Yiğit S, Altındağ A. 2005. A taxonomical study on the zooplankton fauna of Hirfanlı Dam Lake (Kırşehir, Turkey). Gazi University Journal of Science, 18(4):563-567.

Yıldız Ş, Altındağ A, Ergönül MB. 2007. Seasonal fluctuations in the zooplankton composition of a eutrophic lake: Lake Marmara (Manisa, Turkey). Turk J Zool. 31(2):121-126.

YSKY 2012. Yerüstü su kalitesi yönetmeliği (YSKY), 30.11.2012, RG No: 28483, Orman ve Su İşleri Bakanlığı, 28 s. 